\title{
Lu Qi-Keng's problem for intersection of two complex ellipsoids
}

\author{
Tomasz Beberok
}

In this paper We investigate the Lu Qi-Keng problem for intersection of two complex ellipsoids $\left\{z \in \mathbb{C}^{3}:\left|z_{1}\right|^{2}+\left|z_{2}\right|^{q}<1, \quad\left|z_{1}\right|^{2}+\left|z_{3}\right|^{r}<1\right\}$.

Keyword: Lu Qi-Keng problem, Bergman kernel, Routh-Hurwitz theorem

AMS Subject Classifications: 32A25; 33D70

\section{Introduction}

In 1921, S. Bergman introduced a kernel function, which is now known as the Bergman kernel function. It is well known that there exists a unique Bergman kernel function for each bounded domain in $\mathbb{C}^{n}$. Computation of the Bergman kernel function by explicit formulas is an important research direction in several complex variables. Let $D$ be a bounded domain in $\mathbb{C}^{n}$. The Bergman space $L_{a}^{2}(D)$ is the space of all square integrable holomorphic functions on $D$. Then the Bergman kernel $K_{D}(z, w)$ is defined [1] by

$$
K_{D}(z, w)=\sum_{j=0}^{\infty} \phi_{j}(z) \overline{\phi_{j}(w)}, \quad(z, w) \in D \times D,
$$

where $\left\{\phi_{j}(\cdot): j=0,1,2, \ldots\right\}$ is a complete orthonormal basis for $L_{a}^{2}(D)$. In [10] Lu Qi-Keng indicated that in many concrete examples of bounded domains, $K_{D}(z, w) \neq 0$ for all $z, w \in D$, and considered the open problem whether the above property is generally true. M. Skwarczynski [12] called this problem Lu Qi-Keng conjecture in 1969 and gave the following definition:

Definition 1.1 A domain $D \subset \mathbb{C}^{n}$ is called a Lu Qi-Keng domain if $K_{D}(z, w) \neq 0$ for all $z, w \in D$.

Obviously, a biholomorphic image of a Lu Qi-Keng domain is a Lu Qi-Keng domain due to the rule of the Bergman kernel transformation between two biholomorphic equivalent domains. A Cartesian product of two Lu Qi-Keng domains is a Lu Qi-Keng domain. If $K_{D} \neq$ const and $D$ is the sum of an increasing sequence of Lu Qi-Keng domains $D_{m}$, then $D$ is a Lu Qi-Keng domain due to the Ramadanov theorem and Hurwitz theorem.

However, it is not always easy to determine whether or not a given domain is Lu Qi-Keng domain. In 1969, M. Skwarczynski [12] gave the first example that the Bergman kernel on an annulus in the complex plane $\Omega=\{r<|z|<1\}$ has zeros if $0<r<e^{-2}$. Since then many counterexamples appeared. In 1996, Harold P. Boas [3] proved that the bounded domains of holomorphy in $\mathbb{C}^{n}$ whose Bergman kernel 
functions are zero-free form a nowhere dense subset (with respect to a variant of the Hausdorff distance) of all bounded domains of holomorphy. Thus, contrary to many expectations, it is the normal situation for the Bergman kernel function of a domain to have zeroes. For more details, see the survey articles [2] and [14].

Among all the known counterexamples, the following complex ellipsoids

$$
\Omega_{m, n}^{(p, q)}:=\left\{(w, z) \in \mathbb{C}^{m} \times \mathbb{C}^{n}:\|w\|_{m}^{2 p}+\|z\|_{n}^{2 q}<1\right\}
$$

were under consideration frequently, where $\|\cdot\|_{m}$ is the standard Hermitian norm in complex Euclidean space and $p, q$ are positive real numbers. For instance, Boas, Fu and Straube [4] proved there exists a strongly convex domain in $\mathbb{C}^{n}(n>2)$ which is not Lu Qi-Keng by computing the kernel for $\Omega_{1,1}^{\left(p, \frac{1}{2}\right)}=\left\{(w, z) \in \mathbb{C}^{2}:|w|^{2 p}+|z|<1\right\}$ has zeroes if and only if $1 / p>2$. Applying the deflation theorem stated in [4 we are led quickly to that $\Omega_{m, n}:=\left\{(w, z) \in \mathbb{C}^{m} \times \mathbb{C}^{n}: \sum_{k=1}^{m}|w|^{2}+\sum_{k=1}^{n}|z|<1\right\}$ is not Lu Qi-Keng iff $m+2 n>4$ [9]. Using this result, Nguyên Viêt Anh [11] exhibited a strongly convex algebraic complete Reinhardt domain which is not Lu Qi-Keng in $\mathbb{C}^{n}$ for any $n \geq 3$.

When $m=q=1$ and $2 p \geq 1$ is not an integer, as an application of a theorem due to M. Engliš [6] and an improvement by B. Chen, Chen [10] proved there exists a constant $n(p)$ depending on $\mathrm{p}$ such that for all $n>n(p)$, the domain $\Omega_{1, n}^{p, 1}=\left\{|w|^{2 p}+\left|z_{1}\right|^{2}+\cdots+\left|z_{n}\right|^{2}<1\right\}$ is not Lu Qi-Keng. A similar argument as in [4] immediately shows that $\left\{|w|^{2 p}+\left|z_{1}\right|+\cdots+\left|z_{n}\right|<1\right\}$ is not Lu Qi-Keng iff $n \geq[n(p) / 2]+1$, where $[n(p) / 2]$ produces the integer part of $n(p) / 2$.

In both the above cases, how zeroes of the Bergman kernel depend on the increasing of the dimension of the vector $z=\left(z_{1}, \ldots, z_{n}\right)$ are described. The purpose of this paper is to consider intersection of two complex ellipsoids $\left\{z=\left(z_{1}, z_{2}, z_{3}\right) \in\right.$ $\left.\mathbb{C}^{3}:\left|z_{1}\right|^{2}+\left|z_{2}\right|^{q}<1, \quad\left|z_{1}\right|^{2}+\left|z_{3}\right|^{r}<1\right\}$.

\section{Main results}

The following is the main theorem of this paper.

Theorem 2.1 (Main Theorem) For any positive real numbers $q$ and $r$ the domain

$$
\left\{z \in \mathbb{C}^{3}:\left|z_{1}\right|^{2}+\left|z_{2}\right|^{q}<1, \quad\left|z_{1}\right|^{2}+\left|z_{3}\right|^{r}<1\right\}
$$

is a Lu Qi-Keng domain.

\section{Bergman kernel}

For Reinhardt domains it is a standard method for computing the Bergman kernel to use series representation, since we can choose $\phi_{\alpha}(z)=\frac{z^{\alpha}}{\left\|z^{\alpha}\right\|}$. Put $\Phi_{\alpha}(\zeta)=$ $z_{1}^{\alpha_{1}} z_{2}^{\alpha_{2}} z_{3}^{\alpha_{3}}$. It is well known, that function $f$ holomorphic in a Reinhardt domain $D \subset \mathbb{C}^{n}$ has a "global" expansion into a Laurent series $f(z)=\sum_{\alpha \in \mathbb{Z}^{n}} a_{\alpha} z^{\alpha}, z \in D$ (see Proposition 1.7.15 (c) in [8]). Moreover if $D \cap\left(\mathbb{C}^{j-1} \times\{0\} \times \mathbb{C}^{n-j}\right) \neq \emptyset$, $j=1, \ldots, n$ then $a_{\alpha}=0$ for $\alpha \in \mathbb{Z}^{n} \backslash \mathbb{Z}_{+}^{n}$ (see Proposition 1.6.5 (c) in [8]). 
Therefore $\left\{\Phi_{\alpha}\right\}$ such that each $\alpha_{i} \geq 0$ is a complete orthogonal set for $L^{2}\left(D_{q, r}^{p}\right)$, where

$$
D_{q, r}^{p}:=\left\{z \in \mathbb{C}^{3}:\left|z_{1}\right|^{p}+\left|z_{2}\right|^{q}<1, \quad\left|z_{1}\right|^{p}+\left|z_{3}\right|^{r}<1\right\} .
$$

Proposition 3.1 Let $\alpha_{i} \in \mathbb{Z}_{+}$for $i=1,2,3$. Then, we have

$$
\left\|z_{1}^{\alpha_{1}} z_{2}^{\alpha_{2}} z_{3}^{\alpha_{3}}\right\|_{L^{2}\left(D_{q, r}^{2}\right)}^{2}=\frac{\pi^{3} \Gamma\left(\alpha_{1}+1\right) \Gamma\left(\frac{2 \alpha_{2}+2}{q}+\frac{2 \alpha_{3}+2}{r}+1\right)}{\left(\alpha_{2}+1\right)\left(\alpha_{3}+1\right) \Gamma\left(\frac{2 \alpha_{2}+2}{q}+\frac{2 \alpha_{3}+2}{r}+\alpha_{1}+2\right)}
$$

Proof.

$$
\left\|z_{1}^{\alpha_{1}} z_{2}^{\alpha_{2}} z_{3}^{\alpha_{3}}\right\|_{L^{2}\left(D_{q, r}^{2}\right)}^{2}=\int_{D_{q, r}^{2}}\left|z_{1}\right|^{2 \alpha_{1}}\left|z_{2}\right|^{2 \alpha_{2}}\left|z_{3}\right|^{2 \alpha_{3}} d V(z)
$$

we introduce polar coordinate in each variable by putting $z_{1}=r_{1} e^{i \theta_{1}}, z_{2}=r_{2} e^{i \theta_{2}}$, $z_{3}=r_{3} e^{i \theta_{3}}$. After doing so, and integrating out the angular variables we have

$$
(2 \pi)^{3} \int_{0}^{1} \int_{0}^{\left(1-r_{1}^{2}\right)^{1 / q}} \int_{0}^{\left(1-r_{1}^{2}\right)^{1 / r}} r_{1}^{2 \alpha_{1}+1} r_{2}^{2 \alpha_{2}+1} r_{3}^{2 \alpha_{3}+1} d r_{1} d r_{2} d r_{3}
$$

Integrating out of $r_{2}$ and $r_{3}$ variables, we obtain

$$
\frac{(2 \pi)^{3}}{\left(2 \alpha_{2}+2\right)\left(2 \alpha_{3}+2\right)} \int_{0}^{1} r_{1}^{2 \alpha_{1}+1}\left(1-r_{1}^{2}\right)^{\frac{2 \alpha_{2}+2}{q}+\frac{2 \alpha_{3}+2}{r}} d r_{1}
$$

After little calculation using well known fact

$$
\int_{0}^{1} x^{a}\left(1-x^{p}\right)^{b} d x=\frac{\Gamma((a+1) / p) \Gamma(b+1)}{p \Gamma((a+1) / p+b+1)},
$$

we obtain desired result.

Now we discuss the Bergman kernel for $D_{q, r}^{2}$.

Theorem 3.1 The Bergman kernel for

$$
\left\{z \in \mathbb{C}^{3}:\left|z_{1}\right|^{2}+\left|z_{2}\right|^{q}<1, \quad\left|z_{1}\right|^{2}+\left|z_{3}\right|^{r}<1\right\}
$$

is given by

$$
\begin{aligned}
& K_{D_{q, r}^{2}}\left(\left(z_{1}, z_{2}, z_{3}\right),\left(w_{1}, w_{2}, w_{3}\right)\right)= \\
& \quad \frac{q r\left(1-\mu_{2}\right)\left(1-\mu_{3}\right)+2 q\left(1-\mu_{2}\right)\left(1+\mu_{3}\right)+2 r\left(1+\mu_{2}\right)\left(1-\mu_{3}\right)}{\pi^{3} q r\left(1-\nu_{1}^{2}\right)^{2+2 / q+2 / r}\left(1-\mu_{2}\right)^{3}\left(1-\mu_{3}\right)^{3}},
\end{aligned}
$$

where $\nu_{1}=z_{1} \bar{w}_{1}, \nu_{2}=z_{2} \bar{w}_{2}, \nu_{3}=z_{3} \bar{w}_{3}$ and $\mu_{2}=\frac{\nu_{2}}{\left(1-\nu_{1}\right)^{2 / q}}, \mu_{3}=\frac{\nu_{3}}{\left(1-\nu_{1}\right)^{2 / r}}$.

\section{Proof.}

By series representation of the Bergman kernel function, we have

$$
\begin{aligned}
& K_{D_{q, r}^{2}}\left(\left(z_{1}, z_{2}, z_{3}\right),\left(w_{1}, w_{2}, w_{3}\right)\right)=\frac{1}{\pi^{3}} \\
& \quad \sum_{\alpha_{1}, \alpha_{2}, \alpha_{3}=0}^{\infty} \frac{\left(\alpha_{2}+1\right)\left(\alpha_{3}+1\right) \Gamma\left(\frac{2 \alpha_{2}+2}{q}+\frac{2 \alpha_{3}+2}{r}+\alpha_{1}+2\right)}{\Gamma\left(\alpha_{1}+1\right) \Gamma\left(\frac{2 \alpha_{2}+2}{q}+\frac{2 \alpha_{3}+2}{r}+1\right)} \nu_{1}^{\alpha_{1}} \nu_{2}^{\alpha_{2}} \nu_{3}^{\alpha_{3}},
\end{aligned}
$$


where $\nu_{1}=z_{1} \bar{w}_{1}, \nu_{2}=z_{2} \bar{w}_{2}, \nu_{3}=z_{3} \bar{w}_{3}$.

Sum out of $\nu_{1}$ variable, we have

$$
\left(\frac{1}{1-\nu_{1}^{2}}\right)^{2+2 / q+2 / r} \sum_{\alpha_{2}, \alpha_{3}=0}^{\infty} \frac{\left(\alpha_{2}+1\right)\left(\alpha_{3}+1\right) \Gamma\left(\frac{2 \alpha_{2}+2}{q}+\frac{2 \alpha_{3}+2}{r}+2\right)}{\pi^{3} \Gamma\left(\frac{2 \alpha_{2}+2}{q}+\frac{2 \alpha_{3}+2}{r}+1\right)} \mu_{2}^{\alpha_{2}} \mu_{3}^{\alpha_{3}},
$$

where $\mu_{2}=\frac{\nu_{2}}{\left(1-\nu_{1}\right)^{2 / q}}, \mu_{3}=\frac{\nu_{3}}{\left(1-\nu_{1}\right)^{2 / r}}$.

Using the identity $\Gamma(a+1)=a \Gamma(a)$, after a little simplification, we obtain

$$
\sum_{\alpha_{2}, \alpha_{3}=0}^{\infty} \frac{\left(\alpha_{2}+1\right)\left(\alpha_{3}+1\right)\left(\frac{2 \alpha_{2}+2}{q}+\frac{2 \alpha_{3}+2}{r}+1\right)}{\pi^{3}\left(1-\nu_{1}^{2}\right)^{2+2 / q+2 / r}} \mu_{2}^{\alpha_{2}} \mu_{3}^{\alpha_{3}}
$$

After a little calculations, we have

$$
\frac{q r\left(1-\mu_{2}\right)\left(1-\mu_{3}\right)+2 q\left(1-\mu_{2}\right)\left(1+\mu_{3}\right)+2 r\left(1+\mu_{2}\right)\left(1-\mu_{3}\right)}{\pi^{3} q r\left(1-\nu_{1}^{2}\right)^{2+2 / q+2 / r}\left(1-\mu_{2}\right)^{3}\left(1-\mu_{3}\right)^{3}} .
$$

\section{Proof of the main theorem}

Note that the zero set is a bi-holomorphic invariant object. Since any point $\left(z_{1}, z_{2}, z_{3}\right) \in D_{q, r}^{2}$ can be mapped equivalently onto the form $\left(0, \widetilde{z_{2}}, \widetilde{z_{3}}\right)$ by following automorphism of the $D_{q, r}^{2}$

$$
D_{q, r}^{2} \ni\left(z_{1}, z_{2}, z_{3}\right) \mapsto\left(\frac{z_{1}-a}{1-\bar{a} z_{1}}, \frac{\left(1-|a|^{2}\right)^{1 / q}}{\left(1-\bar{a} z_{1}\right)^{2 / q}} z_{2}, \frac{\left(1-|a|^{2}\right)^{1 / r}}{\left(1-\bar{a} z_{1}\right)^{2 / r}} z_{3}\right) \in \mathbb{C}^{3} .
$$

Therefore, we need only consider the zeroes restricted to $\mathbb{D} \times \mathbb{D}$, where $\mathbb{D}:=\{z \in$ $\mathbb{C}:|z|<1\}$. Now by Theorem 3.1

$$
\begin{aligned}
& K_{D_{q, r}^{2}}\left(\left(0, z_{2}, z_{3}\right),\left(0, w_{2}, w_{3}\right)\right)= \\
& \quad \frac{q r\left(1-\nu_{2}\right)\left(1-\nu_{3}\right)+2 q\left(1-\nu_{2}\right)\left(1+\nu_{3}\right)+2 r\left(1+\nu_{2}\right)\left(1-\nu_{3}\right)}{\pi^{3} q r\left(1-\nu_{2}\right)^{3}\left(1-\nu_{3}\right)^{3}},
\end{aligned}
$$

where $\nu_{2}=z_{2} \bar{w}_{2}, \nu_{3}=z_{3} \bar{w}_{3}$.

Denote by

$$
F(x, y)=q r(1-x)(1-y)+2 q(1-x)(1+y)+2 r(1+x)(1-y),
$$

then the Bergman kernel $K_{D_{q, r}^{2}}$ is zero free inside $D_{q, r}^{2} \times D_{q, r}^{2}$ if and only if $F(x, y) \neq$ 0 for all $(x, y) \in \mathbb{D} \times \mathbb{D}$.

Let us recall the stability criteria for a real two-variable polynomial

$$
h(s, z)=\sum_{j=0}^{n} \sum_{k=0}^{m} h_{j k} s^{j} z^{k}
$$


where $s, z \in \mathbb{C}$ are complex variables, and for some $j, k$ the coefficients $h_{j k}$ are not both zero. Polynomial $h(s, z)$ satisfies the stability property

$$
h(s, z) \neq 0, \quad(s, z) \in \overline{\mathbb{D}} \times \overline{\mathbb{D}},
$$

where $\overline{\mathbb{D}}$ is the closure of $\mathbb{D}$.

By following Huang [7], one can show that 1 is equivalent to

$$
\begin{aligned}
h(s, 0) & \neq 0, \quad \forall s \in \overline{\mathbb{D}} \\
h\left(e^{i w}, z\right) & \neq 0, \quad \forall z \in \overline{\mathbb{D}} .
\end{aligned}
$$

Condition (2) means that the new polynomial $f(s)=s^{n} h\left(s^{-1}, 0\right)$ has all zeros in the open unit circle $\mathbb{D}$, that is, $f(s)$ is $\mathbb{D}$-stable. To test condition (3), we consider $d(z)=z^{m} h\left(e^{i w}, z^{-1}\right)$ which we write as a polynomial

$$
d(z)=\sum_{k=0}^{m} d_{k} z^{k}
$$

with coefficients $d_{k}=\sum_{j=0}^{n} h_{j, m-k} s^{k}$, and $s=e^{i w}$.

With the polynomial $d(z)$ we associate the Schur-Cohn $m \times m$ matrix $M=\left(d_{j k}\right)$ specified by

$$
d_{j k}=\sum_{l=1}^{j}\left(d_{m-j+l} \bar{d}_{m-k+l}-\bar{d}_{j-l} d_{k-l}\right),
$$

where $j \leq k$. The matrix $M\left(e^{i w}\right)$ is a Hermitian matrix and we define

$$
g\left(e^{i w}\right)=\operatorname{det} M\left(e^{i w}\right),
$$

where $g(\cdot)$ is a self-inversive polynomial.

We state the following (see [13])

Theorem 4.1 A two-variable polynomial $h(s, z)$ has the stability property 1 if and only if

(i) $f(s)$ is $\mathbb{D}$-stable.

(ii) $g(z)$ is $\mathbb{T}$-positive.

(iii) $M(1)$ is positive definite,

where $\mathbb{T}:=\{z \in \mathbb{C}:|z|=1\}$.

It is easy observation, that $D_{q, r}^{2}$ is Lu Qi-Keng domain if and only if polynomial $F(\varepsilon x, \varepsilon y)$ satisfies the stability property 1 for every $\varepsilon \in(0,1)$.

Let $\varepsilon \in(0,1)$, then

$$
\begin{aligned}
F(\varepsilon x, \varepsilon y)= & \left(\varepsilon^{2} q r-2 \varepsilon^{2} q-2 \varepsilon^{2} r\right) x y+q r+2 q+2 r \\
& +(-\varepsilon q r-2 \varepsilon q+2 \varepsilon r) x+(-\varepsilon q r+2 \varepsilon q-2 \varepsilon r) y
\end{aligned}
$$


Now We will consider conditions (i), (ii) and (iii) from Theorem 4.1 for polynomial $F(\varepsilon x, \varepsilon y)$, where $x, y \in \mathbb{C}$ are complex variables.

Condition (i) means, that the polynomial

$$
f(s)=(q r+2 q+2 r) s+2 \varepsilon r-\varepsilon q r-2 \varepsilon q
$$

has all zeros in the open unit circle $\mathbb{D}$, which is equivalent, to state that the following inequalities

$$
-1<\frac{\varepsilon q r+2 \varepsilon q-2 \varepsilon r}{q r+2 q+2 r}<1
$$

holds for every $q>0, r>0$. Simple calculations show that, these inequalities holds for any positive numbers $p$ and $r$.

In our case condition (iii) is included in condtion (ii), so we need only consider condition (ii). To test condition (ii), we consider polynomial

$$
g(z)=A^{2}+B^{2}-C^{2}-D^{2}+(A \cdot B-C \cdot D)(z+\bar{z}),
$$

where $A=q r+2 q+2 r, B=-\varepsilon q r-2 \varepsilon q+2 \varepsilon r, C=-\varepsilon q r+2 \varepsilon q-2 \varepsilon r$, $D=\varepsilon^{2} q r-2 \varepsilon^{2} q-2 \varepsilon^{2} r$.

Positivity of $g(z)$ on $\mathbb{T}$ (which is required by condition (ii) ) means, that following inequality

$$
(A \cdot B-C \cdot D) x>C^{2}+D^{2}-A^{2}-B^{2}
$$

holds for every $q>0, r>0$ and $-1 \leq x \leq 1$. Easy calculation shows that inequality 4 for every $q>0, r>0$ is true in cases when $x=-2$ or $x=2$. Which implies, that 4 is true for every $q>0, r>0$ and $-1 \leq x \leq 1$. This completes the proof of the main theorem.

\section{Additional results}

Now We will consider following domains

$$
D_{q, r}^{1}:=\left\{z \in \mathbb{C}^{3}:\left|z_{1}\right|+\left|z_{2}\right|^{q}<1, \quad\left|z_{1}\right|+\left|z_{3}\right|^{r}<1\right\} .
$$

Similarly as in section 3 , we have

Proposition 5.1 Let $\alpha_{i} \in \mathbb{Z}_{+}$for $i=1,2,3$. Then, we have

$$
\left\|z_{1}^{\alpha_{1}} z_{2}^{\alpha_{2}} z_{3}^{\alpha_{3}}\right\|_{L^{2}\left(D_{q, r}^{1}\right)}^{2}=\frac{\pi^{3} \Gamma\left(2 \alpha_{1}+2\right) \Gamma\left(\frac{2 \alpha_{2}+2}{q}+\frac{2 \alpha_{3}+2}{r}+1\right)}{\left(\alpha_{2}+1\right)\left(\alpha_{3}+1\right) \Gamma\left(\frac{2 \alpha_{2}+2}{q}+\frac{2 \alpha_{3}+2}{r}+2 \alpha_{1}+3\right)} .
$$

By series representation of the Bergman kernel function, we have

$$
\begin{aligned}
& K_{D_{q, r}^{1}}\left(\left(0, z_{2}, z_{3}\right),\left(0, w_{2}, w_{3}\right)\right)= \\
& \quad \frac{1}{2 \pi^{3}} \sum_{\alpha_{2}, \alpha_{3}=0}^{\infty} \frac{\left(\alpha_{2}+1\right)\left(\alpha_{3}+1\right) \Gamma\left(\frac{2 \alpha_{2}+2}{q}+\frac{2 \alpha_{3}+2}{r}+3\right)}{\Gamma(2) \Gamma\left(\frac{2 \alpha_{2}+2}{q}+\frac{2 \alpha_{3}+2}{r}+1\right)} \nu_{2}^{\alpha_{2}} \nu_{3}^{\alpha_{3}},
\end{aligned}
$$


where $\nu_{2}=z_{2} \bar{w}_{2}, \nu_{3}=z_{3} \bar{w}_{3}$.

Using the identity $\Gamma(a+1)=a \Gamma(a)$, after a little calculation, we obtain

$$
\begin{aligned}
& K_{D_{q, r}^{1}}\left(\left(0, z_{2}, z_{3}\right),\left(0, w_{2}, w_{3}\right)\right)=\frac{2 r^{2}\left(\nu_{2}\left(\nu_{2}+4\right)+1\right)\left(1-\nu_{3}\right)^{2}}{\pi^{3} q^{2} r^{2}\left(1-\nu_{2}\right)^{4}\left(1-\nu_{3}\right)^{4}} \\
& +\frac{q^{2}\left(1-\nu_{2}\right)^{2}\left(-2 r^{2} \nu_{3}+(r-2)(r-1) \nu_{3}^{2}+(r+3) r+8 \nu_{3}+2\right)}{\pi^{3} q^{2} r^{2}\left(1-\nu_{2}\right)^{4}\left(1-\nu_{3}\right)^{4}} \\
& \quad-\frac{q r\left(1-\nu_{2}^{2}\right)\left(1-\nu_{3}\right)\left(3 r\left(\nu_{3}-1\right)-4\left(\nu_{3}+1\right)\right)}{\pi^{3} q^{2} r^{2}\left(1-\nu_{2}\right)^{4}\left(1-\nu_{3}\right)^{4}},
\end{aligned}
$$

where $\nu_{2}=z_{2} \bar{w}_{2}, \nu_{3}=z_{3} \bar{w}_{3}$.

Denote by

$$
\begin{aligned}
G(x, y)= & q^{2}(x-1)^{2}\left(-2 r^{2} y+(r-2)(r-1) y^{2}+(r+3) r+8 y+2\right) \\
& -q r\left(x^{2}-1\right)(y-1)(3 r(y-1)-4(y+1)) \\
& +2 r^{2}(x(x+4)+1)(y-1)^{2},
\end{aligned}
$$

then the Bergman kernel $K_{D_{q, r}^{1}}$ has zero inside $D_{q, r}^{1} \times D_{q, r}^{1}$ if polynomial $G(\varepsilon x, \varepsilon y)$ does not satisfy the stability property 1 for some $0<\varepsilon<1$.

Now We will consider conditions (i) from Theorem 4.1 for polynomial $G(\varepsilon x, \varepsilon y)$ in case when $q=r$. If $q=r$, then we have

$$
\begin{aligned}
G(\varepsilon x, \varepsilon y)= & A_{22} x^{2} y^{2}+A_{21} x^{2} y+A_{20} x^{2}+A_{12} x y^{2} \\
& +A_{11} x y+A_{10} x+A_{02} y^{2}+A_{01} y+A_{00},
\end{aligned}
$$

where $A_{22}=\varepsilon^{4}\left(8-6 r+r^{2}\right), A_{21}=\varepsilon^{3}\left(4+6 r-2 r^{2}\right), A_{20}=\varepsilon^{2} r^{2}, A_{12}=A_{21}, A_{11}=$ $\varepsilon^{2} 4\left(r^{2}-32\right), A_{10}=\varepsilon\left(-2 r^{2}-6 r+4\right), A_{20}=A_{02}, A_{10}=A_{01}$, and $A_{00}=r^{2}+6 r+8$.

Condition (i) means, that the polynomial

$$
f(s)=\left(r^{2}+6 r+8\right) s^{2}+\varepsilon\left(-2 r^{2}-6 r+4\right) s+\varepsilon^{2} r^{2}
$$

has all zeros in the open unit circle $\mathbb{D}$, which is equivalent, to state that the transformed polynomial

$$
Q(s)=(s-1)^{2} f\left(\frac{s+1}{s-1}\right)
$$

is Hurwitz stable and $f(1) \neq 0$. It is easy observation, that

$$
f(1)=(1-\varepsilon)^{2} r^{2}+6(1-\varepsilon) r+8+4 \varepsilon>0
$$

if $0<\varepsilon<1$ and $r>0$. Polynomial $Q(s)$ is Hurwitz stable if and only if (see [15] for details)

$$
\frac{2\left(1-\varepsilon^{2}\right) r^{2}+12 r+16}{(1-\varepsilon)^{2} r^{2}+6(1-\varepsilon) r+8+4 \varepsilon}>0
$$

and

$$
\frac{(1+\varepsilon)^{2} r^{2}+6(1+\varepsilon) r+8-4 \varepsilon}{(1-\varepsilon)^{2} r^{2}+6(1-\varepsilon) r+8+4 \varepsilon}>0
$$


which is true when $0<\varepsilon<1$ and $r>0$. Hence $f(s)$ is $\mathbb{D}$-stable.

To test condition (ii), we consider polynomial

$$
\begin{aligned}
d(z)= & \left(A_{00}+A_{10} t+A_{20} t^{2}\right) z^{2}+\left(A_{01}+A_{11} t+A_{21} t^{2}\right) z \\
& +A_{02}+A_{12} t+A_{22} t^{2}
\end{aligned}
$$

where $t=e^{i w}$. With the polynomial $d(z)$ we associate the Schur-Cohn $2 \times 2$ matrix

$$
M(t)=\left[\begin{array}{ll}
d_{2} \bar{d}_{2}-d_{0} \bar{d}_{0} & d_{2} \bar{d}_{1}-\bar{d}_{0} d_{1} \\
\bar{d}_{2} d_{1}-d_{0} \bar{d}_{1} & d_{2} \bar{d}_{2}-d_{0} \bar{d}_{0}
\end{array}\right]
$$

where $d_{2}=A_{00}+A_{10} t+A_{20} t^{2}, d_{1}=A_{01}+A_{11} t+A_{21} t^{2}$ and $d_{0}=A_{02}+A_{12} t+$ $A_{22} t^{2}, t=e^{i w}$. Now we define $g(t)=\operatorname{det} M(t)$. After some calculation for $\varepsilon=1$ (with the help of a computer program Maple or Mathematica), we have

$$
g\left(e^{i w}\right)=27648 r^{10}(\eta-1)^{3}\left(r^{2}(\eta-1)+4\right),
$$

where $\eta=\cos w$. It is easy to see that for every $r>0$ there exist $\eta<1$ such that $g\left(e^{i w}\right)<0$. Hence there exist $1>\varepsilon>0$, such that polynomial $G(\varepsilon x, \varepsilon y)$ does not satisfy the stability property 1. Therefore the Bergman kernel function for $K_{D_{r, r}^{1}}$ is not zero free. As a consequence of above consideration, we have following proposition

Proposition 5.2 For any $r>0$, domain $D_{r, r}^{1}$ defined by

$$
D_{r, r}^{1}:=\left\{z=\left(z_{1}, z_{2}, z_{3}\right) \in \mathbb{C}^{3}:\left|z_{1}\right|+\left|z_{2}\right|^{r}<1, \quad\left|z_{1}\right|+\left|z_{3}\right|^{r}<1\right\}
$$

is not Lu Qi-Keng.

\section{Some remarks}

As stated in Introduction, Bergman kernel for $\Omega_{1,1}^{(p, 1 / 2)}=\left\{(w, z) \in \mathbb{C} \times \mathbb{C}:|w|^{2 p}+\right.$ $|z|<1\}$ has zeroes if and only if $1 / p>2$. Moreover it is well known fact, that the Thullen domain $\Omega_{1,1}^{(p, 1)}=\left\{(w, z) \in \mathbb{C} \times \mathbb{C}:|w|^{2 p}+|z|^{2}<1\right\}$ is a Lu Qi-Keng domain for $p>0$.

In view of the Proposition 5.2 and Theorem 2.1, we can ask the following question: Is there a relationship between the existence of zeros of the Bergman kernel function for domains

$$
\begin{array}{r}
\left\{z \in \mathbb{C}^{3}:\left|z_{1}\right|^{p}+\left|z_{2}\right|^{q}<1, \quad\left|z_{1}\right|^{p}+\left|z_{3}\right|^{q}<1\right\} \\
\text { and }\left\{z \in \mathbb{C}^{2}:\left|z_{1}\right|^{p}+\left|z_{2}\right|^{q}<1\right\} ?
\end{array}
$$

Through Proposition 5.2 we know, that existence of zeros of the Bergman kernel function for $\left\{z \in \mathbb{C}^{3}:\left|z_{1}\right|^{p}+\left|z_{2}\right|^{q}<1, \quad\left|z_{1}\right|^{p}+\left|z_{3}\right|^{q}<1\right\}$ does not imply existence of zeros of the Bergman kernel function for $\left\{z \in \mathbb{C}^{2}:\left|z_{1}\right|^{p}+\left|z_{2}\right|^{q}<1\right\}$ in general. It is interesting question whether the converse is true? 


\section{References}

[1] S. Bergman, Zur Theorie von pseudokonformen Abbildungen, Mat. Sb. (N.S.) 1(43) (1) (1936), 79-96.

[2] H.P. Boas, Lu Qi-Keng's problem, J. Korean Math. Soc., 37(2) (2000), 253267.

[3] H.P. Boas, The Lu Qi-Keng conjecture fails generically, Proc. Amer. Math. Soc. 124(7) (1996), 2021-2027.

[4] H.P. Boas, S. Fu, E.J. Straube, The Bergman kernel function: explicit formulas and zeroes, Proc. Amer. Math. Soc., 127(3) (1999), 805-811.

[5] B. Chen, Weighted Bergman kernel: Asymptotic behavior, applications and comparison results, Studia Math., 174 (2006), 111-130.

[6] M. Engliš, Zeroes of the Bergman kernel of Hartogs domains, Comment. Math. Univ. Carolin., 41 (2000), 199-202.

[7] T.S. Huang, Stability of two-dimensional recursive filters, IEEE Transactions on Audio and Electroacustics, 20 (1972), 158-163.

[8] M. Jarnicki, P. Pflug, First steps in several complex variables: Reinhardt domains, European Mathematical Society, 2008

[9] M. Jarnicki, P. Pflug, Invariant Distances and Metrics in Complex Analysis, Walter de Gruyter, 1993.

[10] L. Qi-Keng, On Kähler manifolds with constant curvature, Chinese Math.Acta, 8 (1966), 283-298.

[11] V.A. Nguyên, The Lu Qi-Keng conjecture fails for strongly convex algebraic complete Reinhardt domains in $\mathbb{C}^{n}(n \geq 3)$, Proc. Amer. Math. Soc. 128 (2000), 1729-1732.

[12] M. Skwarczynski, The distance in theory of pseu-conformal transformations and the Lu Qi-Keng conjecture. Proc. Amer. Math. Soc., 22 (1969), 305-310.

[13] D. D. Šiljak, D. M. Stipanović, Stability of Interval Two-Variable Polynomials and Quasipolynomials via Positivity, Lecture Notes in Control and Information Science, 312 (2005), 165-177.

[14] Y. Weiping, Zeroes of Bergman kernel functions on bounded domains in $\mathbb{C}^{n}$, Adv. Math. (China), 37 (2008) 1-14.

[15] L. Zhang, W. Yin, Lu Qi-Keng's problem on some complex ellipsoids, J. Math. Anal. Appl., 357 (2009), 364-370.

Tomasz Beberok

Department of Applied Mathematics

University of Agriculture in Krakow

ul. Balicka 253c, 30-198 Krakow, Poland

email: tbeberok@ar.krakow.pl 